\title{
Editorial
}

\section{Fundamental Theories and Practice in Service Robotics}

\author{
ShaoPing Bai, ${ }^{1}$ DeZhen Song, ${ }^{2}$ JiZhong Xiao, ${ }^{3}$ Trung Dung Ngo, ${ }^{4}$ and YongSheng $\mathrm{Ou}^{5}$ \\ ${ }^{1}$ Department of Mechanical and Manufacturing Engineering, Aalborg University, 9220 Aalborg, Denmark \\ ${ }^{2}$ Department of Computer Science and Engineering, Texas A\&M University, TAMU 3112, College Station, TX 77843-3112, USA \\ ${ }^{3}$ Department of Electrical Engineering, The City College, City University of New York, New York City, NY 10031, USA \\ ${ }^{4}$ The More-Than-One Robotics Laboratory, Faculty of Science, University of Brunei Darussalam, \\ Bandar Seri Begawan BE1410, Brunei Darussalam \\ ${ }^{5}$ Shenzhen Institutes of Advanced Technology, Chinese Academy of Sciences, Shenzhen, Guangdong 518055, China
}

Correspondence should be addressed to YongSheng Ou; ys.ou@siat.ac.cn

Received 21 June 2015; Accepted 21 June 2015

Copyright (C) 2015 ShaoPing Bai et al. This is an open access article distributed under the Creative Commons Attribution License, which permits unrestricted use, distribution, and reproduction in any medium, provided the original work is properly cited.

Service robotics is becoming an active research area of robotics in the 21st century. Service robots, such as iRobot, HAL, and CareBot, aim at assisting human in professional and domestic environments. Novel service robots are hightech products resulting from synthesizing the most innovative research outcomes of mechanical design, dynamics, sensing, perception, and cognition science, and control engineering. The complexity of system integration requires fundamental and advance knowledge of mathematics for system modeling and representation.

This special issue addresses in fundamental and advanced mathematical modeling analysis and development of service robots. The latest research results in the field of service robots are collected in this special issue including kinematics, dynamics, and design optimization of service robots (L. Bai et al.), vision-based SLAM (X. Zhu et al.), biologically inspired control of service robots (J. Kwon et al.), and surgical robots (Y. Wang and X. Duan).

$\mathrm{X}$. Zhu et al. address the vision-based simultaneous localization and mapping (SLAM) problem with a semantic approach. They employ a binocular vision to detect the landmark positions and identify their semantic relationships. Then, the semantic observation model is created by transforming the semantic relationships into the semantic metric map which is combined with the traditional topological metric map to improve the accuracy of localization and mapping.
L. Bai et al. present multibody dynamics for the flying and landing oblique impact processes of a bioinspired intermittent hopping robot using the impulse-momentum principle. The dynamics model of the robot that involves the multibody configuration, mass distribution, and friction is solved by the linear complementarity conditions in terms of three impact types: unidirectional sliding impact, viscous impact, and reverse sliding impact. Based on the rules for landing impact, the technical design is accordingly proposed for adjusting the robot attitude during the jump and absorbing the impact energy during the landing.

J. Kwon et al. propose a biologically inspired control scheme for a robotic manipulator. They introduce a parameter tuning algorithm for the biologically inspired control that is capable of handling skillful manipulation of the robotic arm. The humanlike manipulation of the robotic arm is embodied by coupling the joints using networked neural dynamics.

Y. Wang and X. Duan present a three-arm surgical robot system for assisted mandible reconstruction surgery. A spatial registration method is developed for the three-arm robot system to complete the transformation of coordinates between four surgical subsystems consisting of a 3D reconstruction image subsystem, a robotic subsystem, an optical measurement subsystem, and a patient subsystem. A novel mathematical algorithm is developed to optimize and compensate the hand-eye coordination. 
R.-J. Yan et al. present a 3D environment mapping method using B-spline surfaces. Two vertical laser range finders are adopted to provide data sets of the environment, and then those data sets with overlaps are merged using the product of Gaussian probability density functions. Experiments were conducted to validate the $3 \mathrm{D}$ mapping performance.

These papers presented in this special issue serve as a good illustration of the emerging challenges of service robotics, which can be solved by the new mathematical tools. By compiling these papers, we hope to enrich our readers and researchers with new mathematical solutions, novel algorithms, and methods on the relevant problems to advance the research and development of service robots.

ShaoPing Bai DeZhen Song JiZhong Xiao

Trung Dung Ngo YongSheng $\mathrm{Ou}$ 


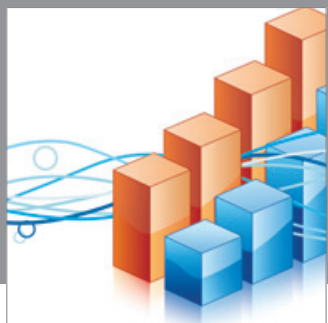

Advances in

Operations Research

mansans

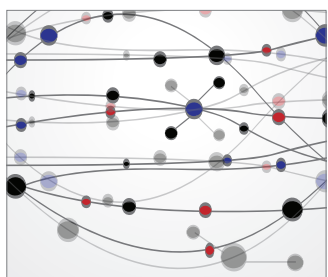

The Scientific World Journal
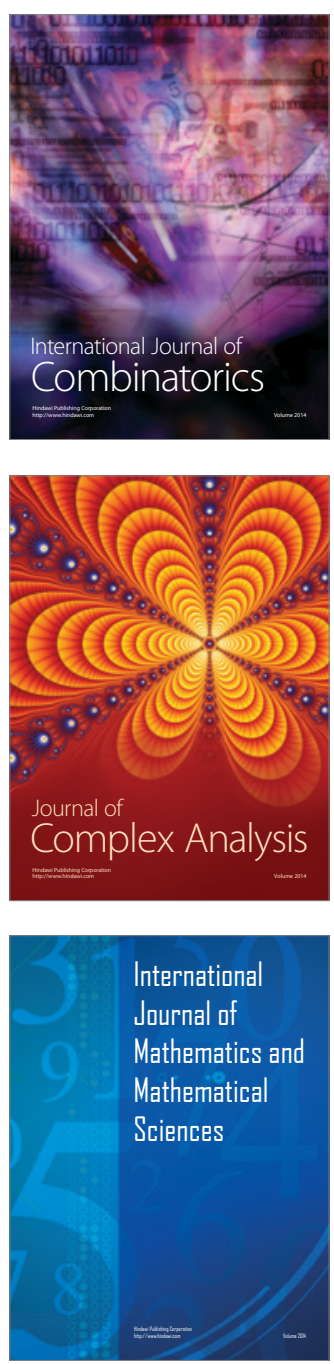
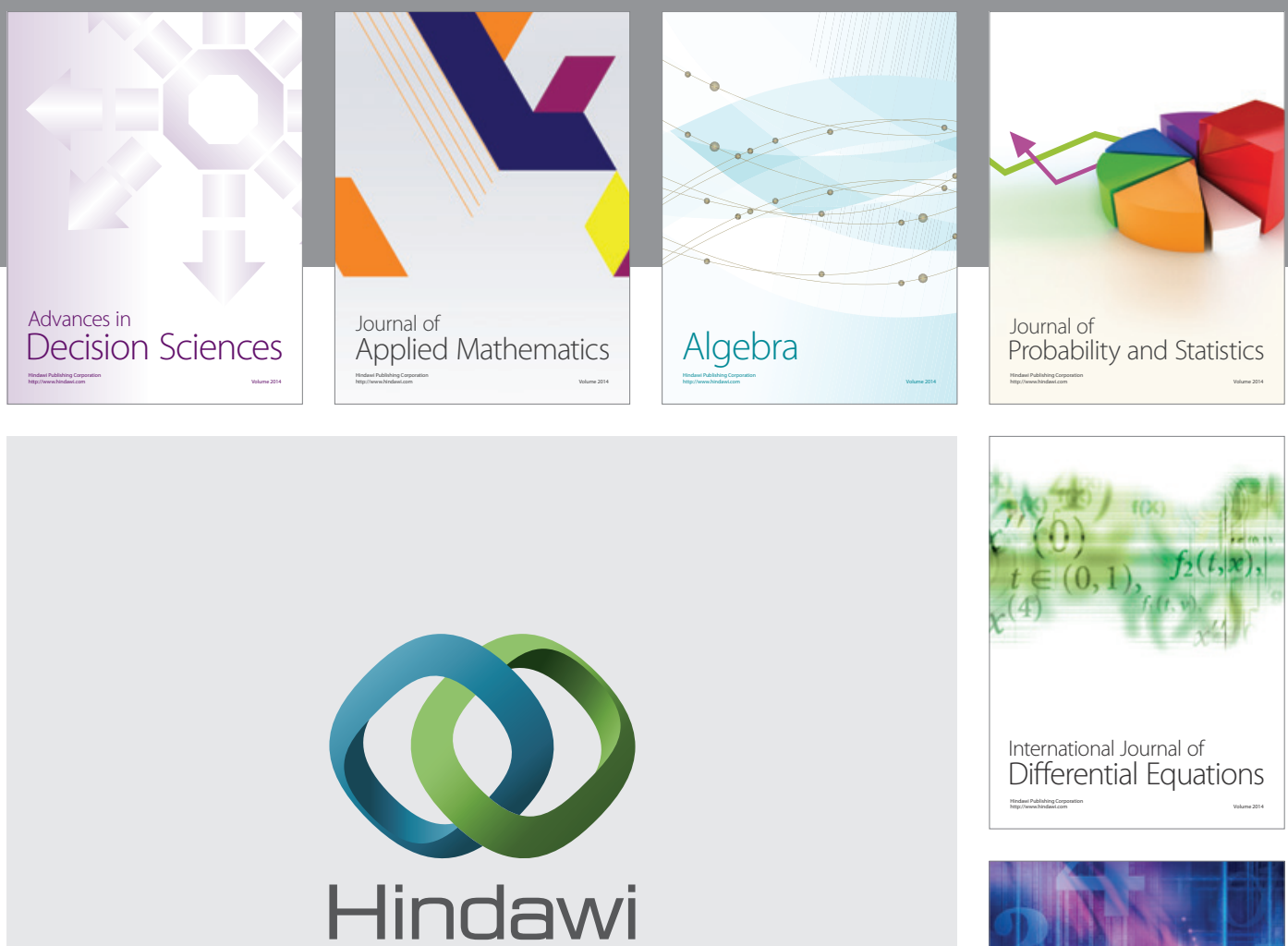

Submit your manuscripts at http://www.hindawi.com
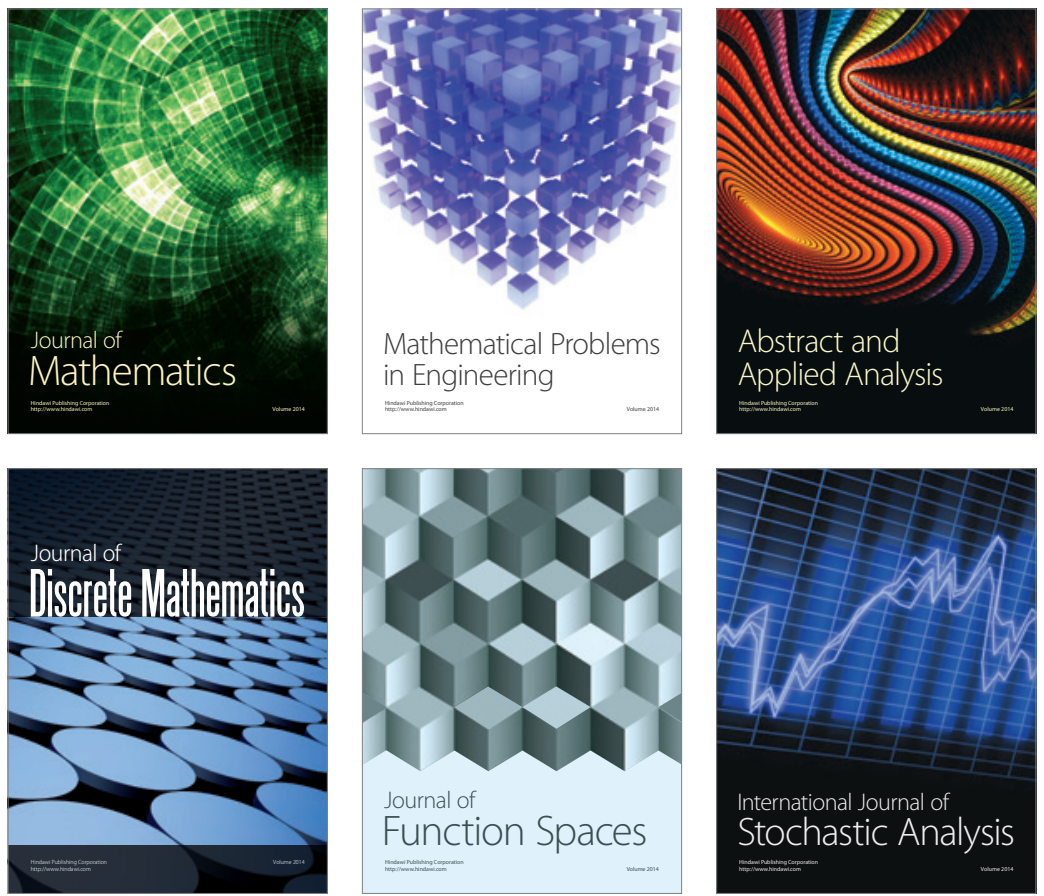

Journal of

Function Spaces

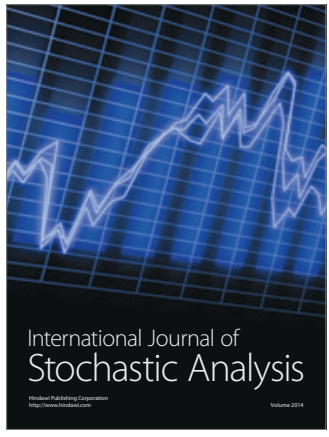

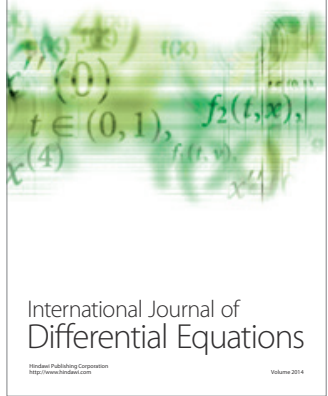
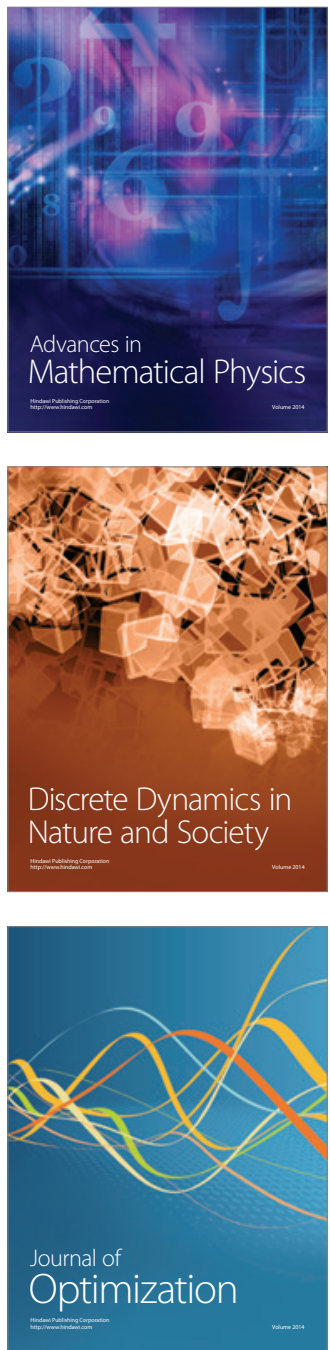